\title{
Osme bond: anisotropic distribution of electron density in action
}

\author{
A. Pizzi ${ }^{1}$, A. Daolio', M. Calabrese ${ }^{1}$, G. Terraneo ${ }^{1}$, A. Frontera, ${ }^{2}$, G. Resnati ${ }^{1}$ \\ 1Department of Chemistry, Materials and Chemical Engineering "Giulio Natta”, Politecnico di Milano, Milano 20131, Italy \\ 2Department of Chemistry, Universitat de les Illes Balears, Crta. de Valldemossa, 07122 Palma de Mallorca (Baleares), Spain
}

andrea.pizzi@polimi.it

The distribution of the electron density on the surface of molecules is typically anisotropic. This leads to regions featuring positive potential that can behave as electrophilic sites in attractive interactions involving regions in surrounding molecules having a negative electrostatic potential. Based on this mindset, a systematic rationalization of intermolecular interactions began in the 1990s, when on the surface of halogen atoms a region of positive electrostatic potential, the so called $\sigma$-hole,[1] was identified and explored as a new tool in supramolecular chemistry.

Analogous $\sigma$-holes were then found on other elements of $p$-block of the periodic table (elements of groups 14,[2] 15,[3] and 16[4]), and at the same time the awareness grew that also chemical interactions can be rationalized as periodic properties. The attractive interactions occurring between these positive regions and nucleophilic sites are now topics of intense research.

Although in adducts involving $d$-block elements the identification of electrophilic and nucleophilic moieties is generally nontrivial, some $\sigma$-holes have been identified on metals in some of these adducts. This is the case, for instance, of positive $\sigma$-holes on the group 11 metals in respective halides [5].

Here, we report how the crystal structures of adducts between nitrogen or oxygen nucleophiles (pyridine or pyridine $N$-oxide derivatives) and osmium tetroxide show short noncovalent $\mathrm{Os} \cdots \mathrm{N} / \mathrm{O}$ contacts. Theoretical evidence suggest that these contacts are $\sigma-$ hole interactions, and that similar adducts of other group 8 elements behaves in a similar way.[6] We propose the term "osme bond" $(\mathrm{OmB}, \mathrm{Om}=\mathrm{Fe}, \mathrm{Ru}, \mathrm{Os},(\mathrm{Hs}))$ for naming the noncovalent interactions wherein group 8 elements behave as electrophile.
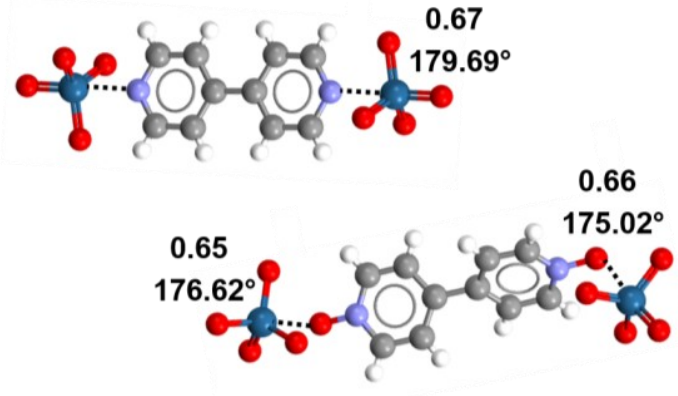

Figure 1. Osme bonded adducts of $\mathrm{OsO}_{4}$ with 4,4'-bipyridine (top) and [4,4'-bipyridine] 1,1'-dioxide (bottom). OmBs are depicted as black dotted lines; Nc and interaction angles are given. White, hydrogen; grey, carbon; blue, nitrogen; red, oxygen; navy, osmium.

[1] Politzer, P., Murray, J. S., Clark, T., Resnati, G. (2017) Phys. Chem. Chem. Phys. 19, 32166.

[2] Daolio, A., Scilabra, P., Terraneo, G., Resnati, G. (2020) Coord. Chem. Rev. 413, 213265.

[3] Mahmudov, K. T., Gurbanov, A. V., Aliyeva, V. A., Resnati, G., Pombeiro, A. J. L. (2020) Coord. Chem. Rev. 418, 213381.

[4] Tripathi, A., Daolio, A., Pizzi, A., Guo, Z., Turner, D. R., Baggioli, A., Famulari, A., Deacon, G. B., Resnati, G., Singh, H. B. (2021) Chem. Asian J. 10.1002/asia.202100545.

[5] Daolio, A., Pizzi, A., Terraneo, G., Ursini, M., Frontera, A., Resnati, G. (2021) Angew. Chemie Int. Ed. $60,14385$.

[6] Daolio. A., Pizzi, A., Calabrese, M., Terraneo, G., Bordignon, S., Frontera, A., Resnati, G. (2021) Angew. Chemie Int. Ed. 10.1002/anie.202107978

Keywords: Osme Bond, Sigma-hole, noncovalent interactions 